\title{
Improved Entropic Threshold based on GLSC Histogram with Varying Similarity Measure
}

\author{
M Seetharama Prasad \\ Singhania University, \\ Pacheri Bari, Rajastan, \\ India-333515
}

\author{
T Divakar \\ LBR College of Engg. \\ Mylavaram \\ India-521230
}

\author{
Dr. L S S Reddy \\ LBR College of Engg. \\ Mylavaram \\ India-521230
}

\begin{abstract}
Image segmentation is a necessary task in computer vision and digital image processing applications, where foreground objects are to be separated from background. Many thresholding techniques are found in literature with their own limitations. The Gray level Spatial Correlation (GLSC) Histogram is used in entropic techniques to decide the threshold. In this paper we propose an improved GLSC Histogram, computed with varying similarity measure $(\boldsymbol{\zeta})$ by considering local and global characteristics, because Yang Xiao et. al. used a constant 4 as the similarity measure by considering the image local properties only, which does not suits for all types of images and probability error is minimized by redistributing the missing probability amount in floating precisions. For low contrast images contrast enhancement is assumed. Experimental results demonstrate a quantitative improvement against existing techniques by calculating the parameter efficiency $\eta$ based on the misclassification error and variations in various y ielding towards ground truth threshold on two dimensional histogram of image.
\end{abstract}

\section{General Terms}

Image processing, entropy

\section{Keywords}

Entropy, GLSC histogram, threshold, image segmentation

\section{INTRODUCTION}

In many computer vision and image processing applications the fundamental task performed on image data is image segmentation, to process the foreground objects in order to explore the features. A well-known technique for image segmentation is thresholding, accuracy of segmentation is depends upon the process which is based on the gray level histogram. It is essential to find the threshold value to group into two well defined non-overlapping subsets. For a well-defined image its histogram has a deep valley between two peaks. To locate the threshold valley region is the best place in bimodal histogram images because both peaks mostly representing the object and back ground pixels but it is not applicable for all types of images.

Many thresholding methods have been found in literature [1-3], still it is an area where the research is alive. Otsu [4] proposed discrimination analysis to find the threshold, failing in many cases where there is no much discrimination in image foreground and back ground intensities. Otsu derived threshold based on several statistical measures on image like mean, variance and standard deviation. Shannon introduced information theory based on concept of entropy [5], Pun [6] first applied this concept of entropy to thresholding, Kapur et.al. proposed a method by improving the work of Pun[7]. This idea is further generalized to Renyi's entropy [8][9] and Tsallis's entropy [10][11]. Yang Xiao et.al further improvised the same work with three dimensional image histogram by considering local properties of image, named gray level spatial correlation Histogram (GLSC)[12] since too more time complexity with the two dimensional histogram[13] approaches. We propose a novel approach by taking the discrimination analysis of Otsu to find spatial correlation measure, and using the same in construction of Three-dimensional GLSC histogram. The missing probability due to floating precision is found in generating the normalized histogram is taken into account and equally distributed.

The remainder of this paper is organized as follows: section 2 describes the existing method; section 3 describes the proposed method, section 4 shows comparative results and improved y ielding of our method and section 5 ends up with conclusion.

\section{EXISTING METHOD}

Spatial correlation histogram differentiates two images in which the frequencies of intensities are exactly same, but they differ in their placements, where two dimensional histograms fail. Therefore different images with identical two-dimensional histogram will result in the same threshold value. To overcome the problem GLSC histogram is constructed by considering the similarity in neighborhood pixels with some adaptive threshold value as similarity measure $(\zeta)$ common to entire image.

Let $f(x, y)$ be the gray level intensity of image at $(x, y)$. $F=\{f(x, y) \mid x \in[1, Q], y \in[1, R]\}$ of size $Q x R$. The gray level set $\{0,1,2, \ldots 255\}$ is considered as $\mathrm{G}$ throughout this paper for convenience. The image GLSC histogram is computed by taking only image local properties into account as follows. Let $\mathrm{g}(\mathrm{x}, \mathrm{y})$ be the similarity count corresponding to pixel of image $f(x, y)$ in $\mathrm{N} \mathrm{X} \mathrm{N} \mathrm{neighborhood,} \mathrm{where} \mathrm{N}$ is any positive odd number in range $[3, \min (\mathrm{Q} / 2, \mathrm{R} / 2)]$.

$$
\begin{aligned}
& \mathrm{g}(\mathrm{x}+1, \mathrm{y}+1)= \\
& \sum_{i=0}^{\mathrm{N}-1} \sum_{j=0}^{N-1} ?(|f(x+1, y+1)-f(x+i, y+j)| \leq \zeta) \\
& \text { Where, ? }(|f(x+1, y+1)-f(x+i, y+j)|)= \\
& \left\{\begin{array}{l}
1 \\
0 \quad \text { otherwise }
\end{array}\right.
\end{aligned}
$$


GLSC histogram is constructed with the correlated probability at different gray level intensities from equations (1) and (2) as follows.

$h(k, m)=P(f(x, y)==k$ and $g(x, y)==m)$

Where, $\mathrm{P}$ is the gray level correlation probability computed for all pixels with intensity $\mathrm{k} \in \mathrm{G}$ with correlation $\mathrm{m} \in\{1,2, . . \mathrm{NXN}\}$ and histogram is normalized [12]. As author discussed in [12] with similarity measure $\zeta=4$ and $\mathrm{N}=3$ the existing method giving its best results.

Kapur, et. al. [7] Entropic function is used as criterion function in estimation of threshold on GLSC histogram instead of 2D gray level histogram with a defined weight for the taken map $\mathrm{N}=3$, and corresponding correlation values from the equations (4) and (5).

$\mathrm{t}^{*}=\operatorname{Arg} \max \phi(\mathrm{t}, \mathrm{N})$

$\phi(t, N)=H_{O}(t, n)+H_{B}(t, n)$

where, $\phi(t, N)$ is the entropic criterion function, $\mathrm{H}_{\mathrm{O}}(\mathrm{t}, \mathrm{n})$ and $\mathrm{H}_{\mathbf{B}}(\mathrm{t}, \mathrm{n})$ are entropies associated with object and background respectively.

\section{PROPOSED METHOD}

The results of the above discussed method are encouraging, introducing spatial correlation into threshold selection conceptually sounding good and the impact of GLSC histogram in entropy computation open new avenues. We observe Yang Xiao et.al. Considered only local properties of the image and stated similarity measure ( 3 ) as constant at 4 producing reasonable results. Still this technique can be extended further, by taking global properties into account along with local properties. In entropy computations the combined probability distributions of both object and background should be a unit, in our observation due to floating precision some amount is missing in both object and back ground probabilities, which should be distributed equally across the probability function $p(k, m)$. The optimum threshold $\mathrm{t} *$ is derived by maximizing the entropic criterion function $\phi(t, N)$ from equations (4), (5) as $\mathrm{t}^{*}=\operatorname{Arg} \max \phi(\mathrm{t}, \mathrm{N})$. From equation (5) $\mathrm{H}_{\mathrm{O}}, \mathrm{H}_{\mathrm{B}}$ are entropies associated with object and back ground distribution

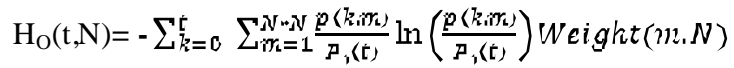

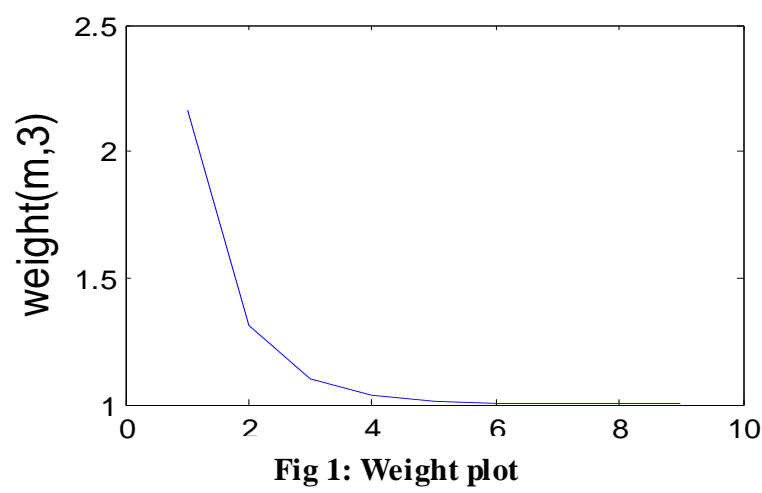

And

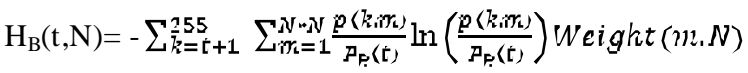

Where, Weight $(m, N)$ is a non linear function applied to GLSC histogram elements for entropic calculation derived as.

$$
\text { Weight }(m, N)=\frac{1+5 \frac{-9 m}{N X N}}{1-5 \frac{-9 m}{N X N}}
$$

Where, $\mathrm{N}$ is any positive odd number in $\operatorname{range}[3, \min (\mathrm{Q} / 2, \mathrm{R} / 2)]$ and $\mathrm{m} \in\{1,2, \ldots . \mathrm{NxN}\}$, Fig (1) shows Weight(m.N) a plot when $\mathrm{N}=3$

\subsection{Missing probability Redistribution}

Probability function $\mathrm{p}(\mathrm{k}, \mathrm{m})$ is calculated with pixel correlation in neighborhood at each gray level intensity is a major component, $P_{\sigma}(t), P_{B}(t)$ are the associated probabilities of object and background used in equations (6) and (7) to calculate the respective entropies $\mathrm{H}_{\mathrm{O}}$ and $\mathrm{H}_{\mathrm{B}}$.

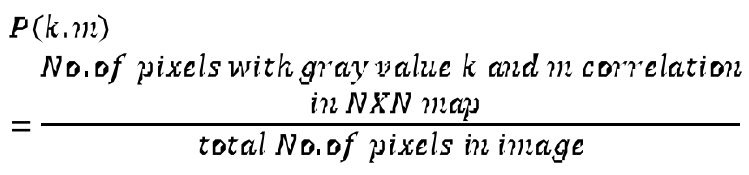

Where,

$\sum_{k=0}^{255} \sum_{m=1}^{N X N} p(k, m)=1$

But, we observe that equation (10) is failing due to the floating precision and there found to be a $\Delta p$ error, which is equally distributed across the $\mathrm{p}(\mathrm{k}, \mathrm{m})$ to improve threshold selection criterion.

$\Delta p=1-\sum_{k=0}^{255} \sum_{m=1}^{N X N} p(k, m)$

Improved probability function $\hat{p}(I, j)=$ 
With the equation (12) we calculate $P_{0}(t)$ and $P_{\bar{B}}(t)$ to threshold image at $\mathrm{t}$ as follows

The two disjoint sets corresponding to object and background are $\hat{G}_{\mathrm{O}}, \hat{G}_{\mathrm{B}}$ are partitioned from set $\hat{G}$ corresponding to entire image with t. the probability distribution associated with object and background are given by

$\left[\frac{\hat{p}(0,1)}{p_{0}(t)}, \ldots \frac{\hat{p}(0, N X N)}{p_{0}(t)}, \frac{\hat{p}(1.1)}{p_{0}(t)} \ldots \frac{\hat{p}(t, N X N)}{P_{0}(t)}\right]$

$\left[\frac{\hat{p}(t+1,1)}{P_{B}(t)}, \ldots \frac{\hat{p}(t+1, N X N)}{P_{B}(t)}, \frac{\hat{p}(t+2,1)}{P_{B}(t)} \ldots \frac{\hat{p}(255, N X N)}{P_{B}(t)}\right]$

Where, $\mathrm{P}_{\mathrm{A}}(\mathrm{t})=\sum_{k=0}^{t} \sum_{m=1}^{N X N} \hat{p}(k, m)$ and $\mathrm{P}_{\mathrm{B}}(\mathrm{t})=1-\mathrm{P}_{\mathrm{A}}(\mathrm{t})$

\subsection{GLSC Histogram with varying ?}

We construct GLSC Histogram with varying similarity measure $(\zeta)$ by taking local and global properties of image to rationalize the process, where as Yang Xiao et. al. suggested a constant value 4 . We follow a procedure to calculate $\zeta$ for every NXN map, with the help of Otsu's discrimination analysis to measure image global and local characteristic $C_{g}$ and $C_{l}$ respectively.

$$
\zeta=2 *\left|C_{g}-C_{1}\right|
$$

whole and local NXN map, when compared with the other statistical methods such as mean, variance and standard deviation. The GLSC histogram using the new $\zeta$ with equations (1) and (2) is computed.

Fig 2.(a) shows 'cameraman.tif' for which 2D, GLSC and Improved GLSC Histograms are computed and shown in fig 2.(b),2.(c) and 2.(d) respectively. In 2D histogram the two peaks represents object and background, in GLSC histogram the same are represented in $3 \mathrm{D}$, which gives the information about spatial correlation. But it fails to identify the ambiguous region, which is well defined in improved GLSC histogram and it offers profound choice to maximize entropy based criterion function. We observe from these two histograms more probability distribution across the image spectrum in our method than existing one, hence it helps Shannon's entropy to obtain optimal threshold $\mathrm{t}^{*}$.

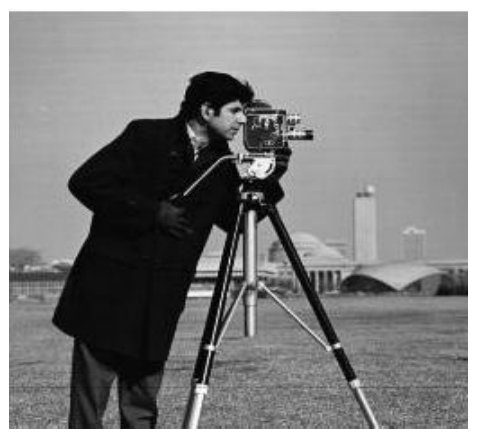

a. Camera man

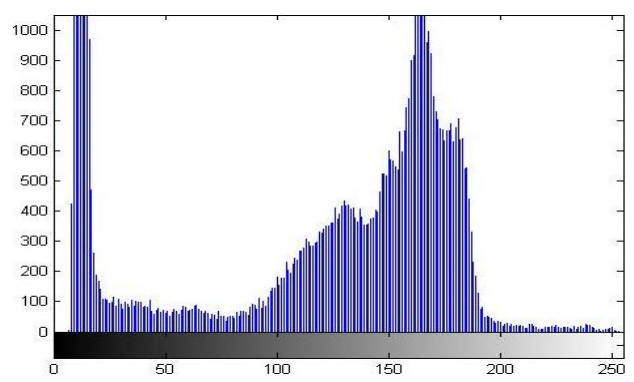

b. Gray level histogram

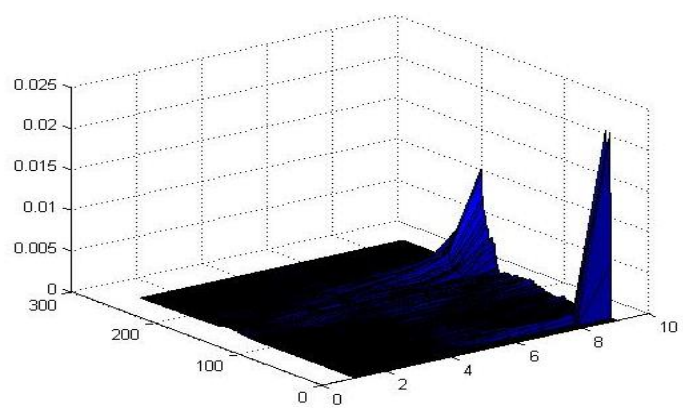

c. GLSC Histogram (constant $\zeta=4$ )

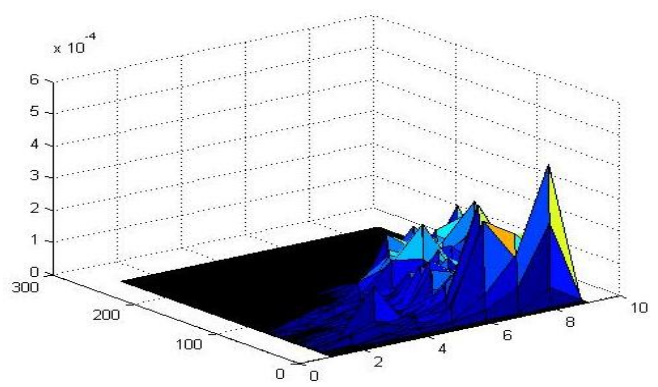

d. Improved GLSC Histogram ( varying $\zeta$ )

Fig 2: Cameraman image, its gray level, GLSC and improved GLSC histograms 

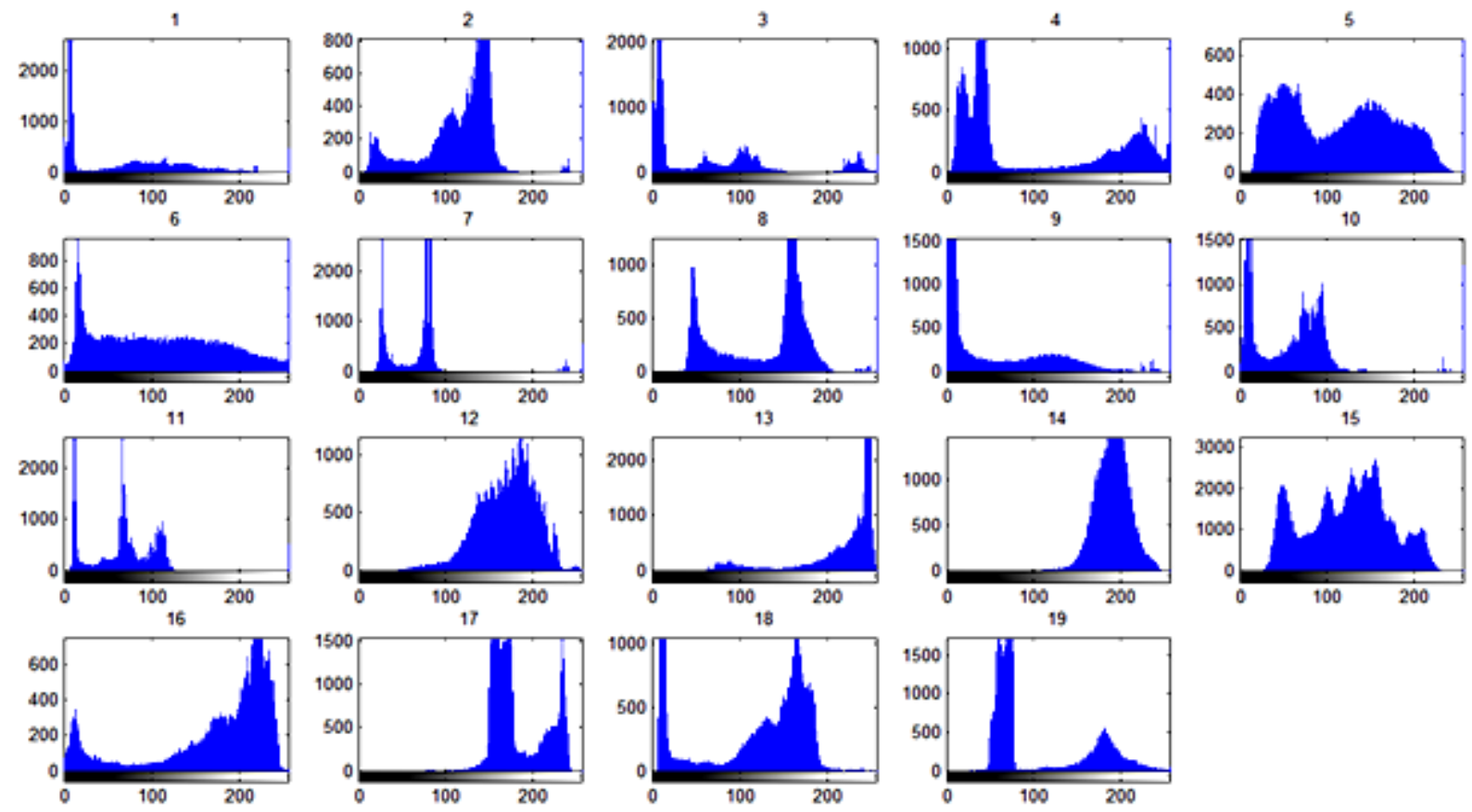

Fig 3: Histograms of image set shows various histogram models
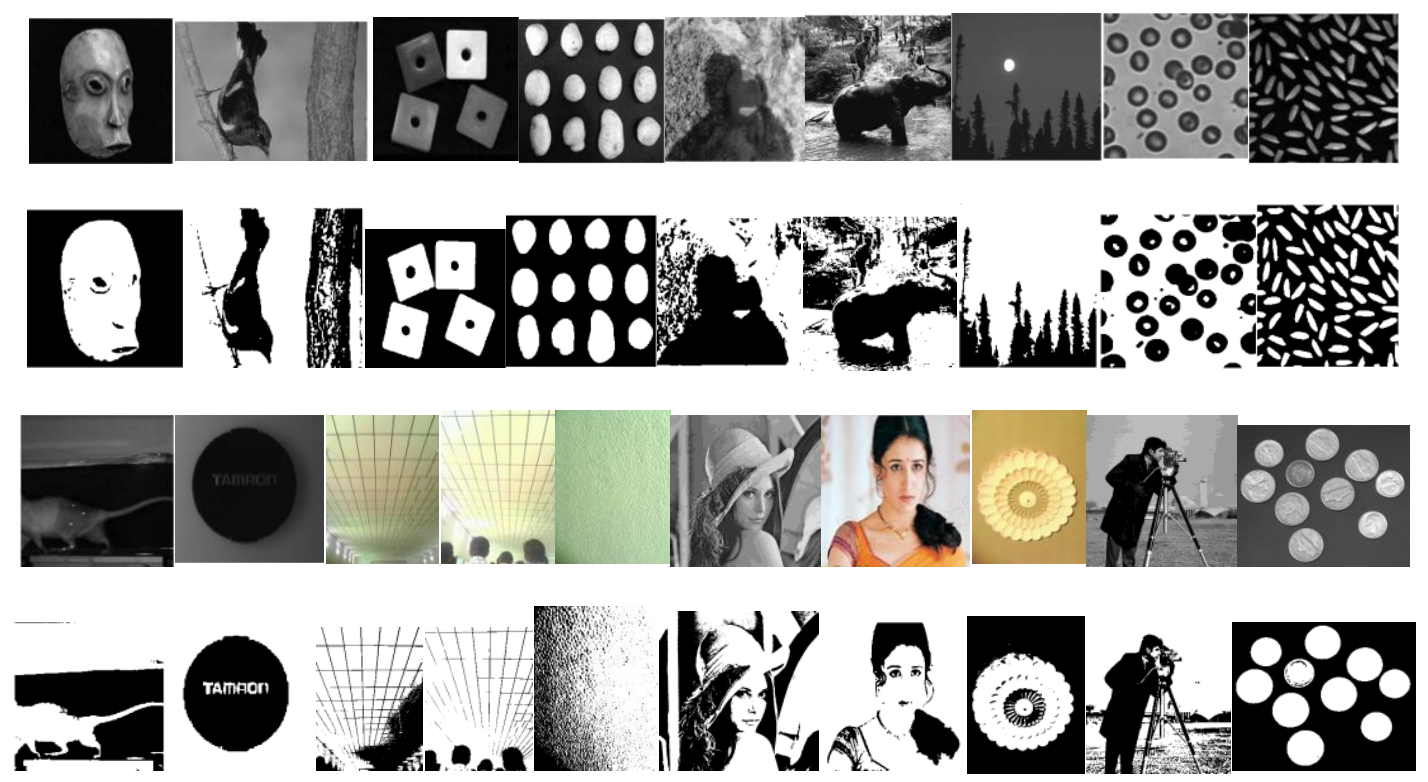

Fig 4: Image set and their corresponding ground-truth possible threshold BW image set

\section{RESULTS AND DISCUSSIONS}

To illustrate the performance of the proposed methodology we consider 19 images as an image set having similar and dissimilar gray level histogram characteristics, varying from uni-model to multimodal as shown in fig 3 and gold standard ground truth images are generated manually to measure a parameter efficiency $(\eta)$ based on misclassification error[2].
Thus

$$
\eta=\frac{\left|M M G_{\mathbf{0}} \cap I M G_{\mathbf{r}}\right|}{|\operatorname{IMG}|} \times 100
$$

Where, $\mathrm{IMG}_{\mathrm{O}}, \mathrm{IMG}_{\mathrm{T}}$ are gold standard image and resultant image respectively and $|*|$ is the Cartesian Number of the set gives number of pixels. This $\eta$ would be 0 for absolutely dissimilar and 100 for exactly similar image as result. Fig 4 
shows original image set and their possible gold standard threshold image set.

From the experiments for each image we obtain four threshold values from different methods including Proposed, GLSCH, OTSU's and Kapur et. al. entropy as in TABLE 1. These thresholds are compared with assumed gold standard thresholds. Fig 5 confirms a variation in threshold in above said methods on histogram range for image set consider. Efficiency $(\eta)$ is calculated for each technique on image set with equation 16. A mean $(\mu)$ and standard deviation $(\sigma)$ are calculated on efficiency in order to show the effectiveness of the proposed and other methods as in TABLE 2. A mean 97.33 and standard deviation 3.19 is obtained from the proposed method which confirms the qualitative improvement over the existing methods. Fig 7 shows threshold results obtained by various techniques.

TABLE 1: Thresholds for various images obtained by different techniques

\begin{tabular}{|c|c|c|c|c|c|c|}
\hline S.NO & IMAGE & GROUNDTROUTH & PROPOSED & GLSCH & OTSU & ENTROPY \\
\hline 1 & Scull & 35 & 49 & 7 & 64 & 6 \\
\hline 2 & Bird & 113 & 124 & 137 & 119 & 131 \\
\hline 3 & Blocks & 46 & 53 & 8 & 155 & 11 \\
\hline 4 & Potatoes & 76 & 85 & 40 & 124 & 44 \\
\hline 5 & Shadow & 123 & 118 & 109 & 126 & 119 \\
\hline 6 & Forest & 96 & 113 & 32 & 122 & 96 \\
\hline 7 & Trees & 57 & 59 & 80 & 153 & 84 \\
\hline 8 & Blood cells & 131 & 132 & 157 & 119 & 156 \\
\hline 9 & Rice & 58 & 51 & 7 & 75 & 11 \\
\hline 10 & Animal & 35 & 43 & 11 & 99 & 54 \\
\hline 11 & Emblem & 25 & 49 & 12 & 80 & 62 \\
\hline 12 & Roof & 151 & 162 & 178 & 171 & 174 \\
\hline 13 & Roof heads & 179 & 174 & 244 & 183 & 256 \\
\hline 14 & Sheet & 180 & 175 & 190 & 186 & 256 \\
\hline 15 & Lena & 123 & 123 & 130 & 133 & 129 \\
\hline 16 & Anshu & 136 & 124 & 212 & 119 & 201 \\
\hline 17 & Wall flower & 181 & 181 & 166 & 197 & 256 \\
\hline 18 & cameraman & 117 & 118 & 132 & 83 & 144 \\
\hline 19 & Coins & 107 & 114 & 71 & 137 & 73 \\
\hline
\end{tabular}

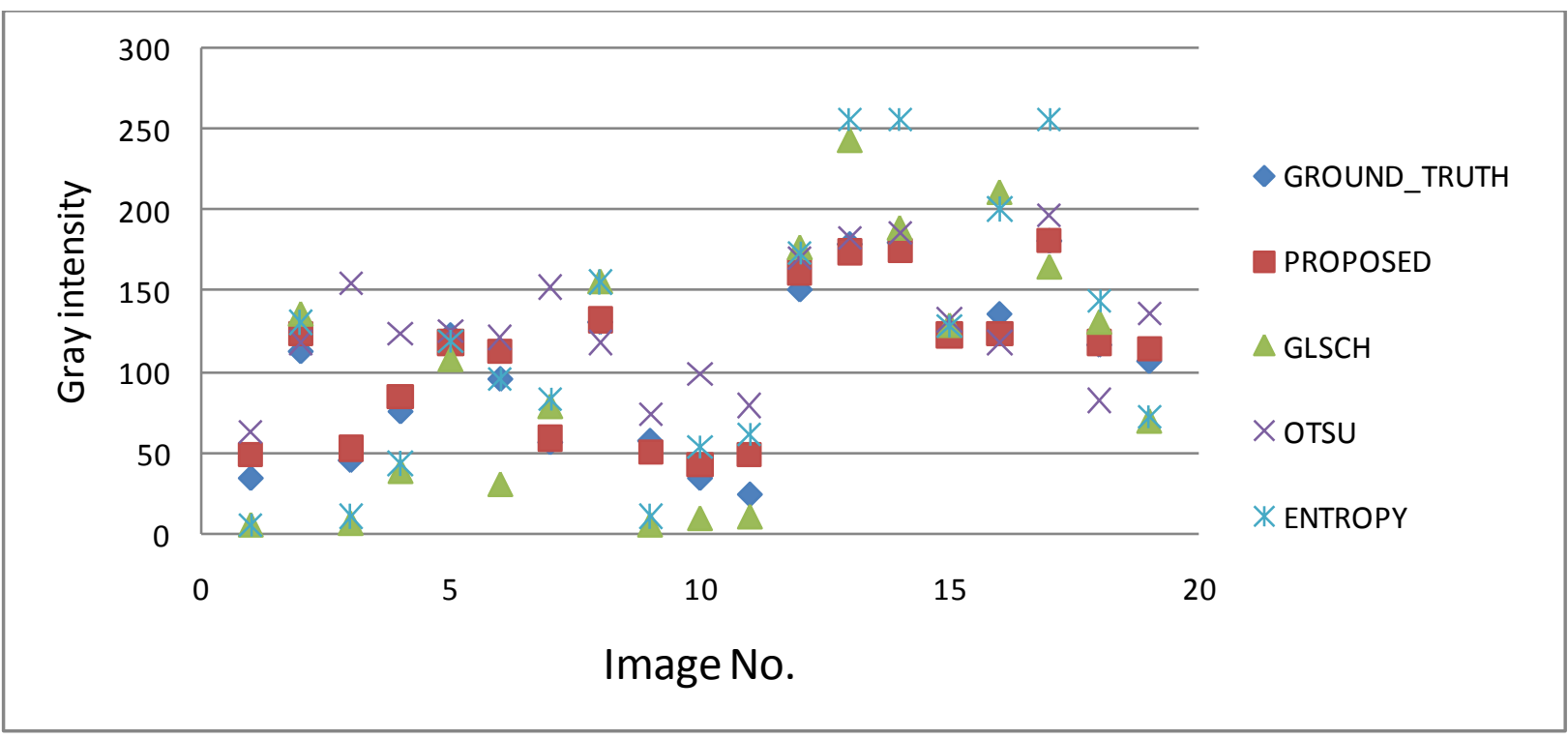

Fig 5: Comparison of thresholds for various images obtained by different techniques 


\section{CONCLUSION}

In this paper a segmentation approach based on improved GLSC histogram is presented, by deriving varying similarity measure and probability error redistribution. The main difference between our work and yang Xiao's proposition is in construction of GLSC Histogram. The nonlinear characteristics of the image which are based on human perception are considered in computing correlation matrix $\mathrm{g}(\mathrm{x}, \mathrm{y})$. GLSC Histogram possesses some advantage over 2D histogram. However, with constant $\boldsymbol{\zeta}$ the process suffers under the conditions where different background brightness intensities effecting the object characteristics. Computer vision system doesn't yield the same perception response to absolute brightness difference. Hence we derived a variable similarity measure $\zeta$ using statistical approach. Efficiency of threshold selection is demonstrated with experimental results. We assume a reasonable contrast enhancement for low contrast images. Performance evolution is carried out with the help of misclassification error on proposed work, GLSC, Otsu's and Kapur's entropy methods. As future work, Ultra fuzziness a fuzzy measure of type II fuzzy set is used instead of Shannon's entropy while computing the optimal threshold.

Table 2: Efficiency of various threshold techniques

\begin{tabular}{|c|c|c|c|c|c|}
\hline S.NO & IMAGE & PROPOSED & GLSCH & OTSU & ENTROPY \\
\hline 1 & Scull & 98.69 & 61.67 & 96.50 & 44.04 \\
\hline 2 & Bird & 92.23 & 76.36 & 96.13 & 84.85 \\
\hline 3 & Blocks & 99.12 & 52.79 & 72.00 & 86.80 \\
\hline 4 & Potatoes & 99.52 & 77.32 & 97.39 & 88.70 \\
\hline 5 & Shadow & 97.85 & 94.62 & 98.71 & 98.29 \\
\hline 6 & Forest & 93.62 & 74.62 & 90.23 & 100.00 \\
\hline 7 & Trees & 99.60 & 68.14 & 28.29 & 33.27 \\
\hline 8 & Blood cells & 99.82 & 84.21 & 97.90 & 86.85 \\
\hline 9 & Rice & 98.62 & 61.81 & 96.67 & 81.32 \\
\hline 10 & Animal & 97.92 & 78.50 & 52.29 & 94.24 \\
\hline 11 & Emblem & 94.22 & 72.57 & 66.46 & 89.47 \\
\hline 12 & Roof & 89.91 & 72.88 & 80.52 & 77.48 \\
\hline 13 & Roof heads & 99.24 & 58.38 & 99.33 & 12.41 \\
\hline 14 & Sheet & 92.16 & 81.25 & 89.21 & 28.88 \\
\hline 15 & Lena & 100.00 & 94.06 & 91.44 & 95.02 \\
\hline 16 & Anshu & 97.67 & 60.29 & 96.92 & 71.04 \\
\hline 17 & Wall flower & 100.00 & 75.55 & 95.56 & 64.84 \\
\hline 18 & Cameraman & 99.55 & 91.68 & 91.50 & 84.69 \\
\hline 19 & Coins & 99.56 & 70.80 & 97.86 & 81.18 \\
\hline & Mean $(\mu)$ & 97.33 & 74.08 & 86.05 & 73.86 \\
& STD $(\sigma)$ & 3.19 & 11.94 & 18.93 & 25.53 \\
\hline
\end{tabular}

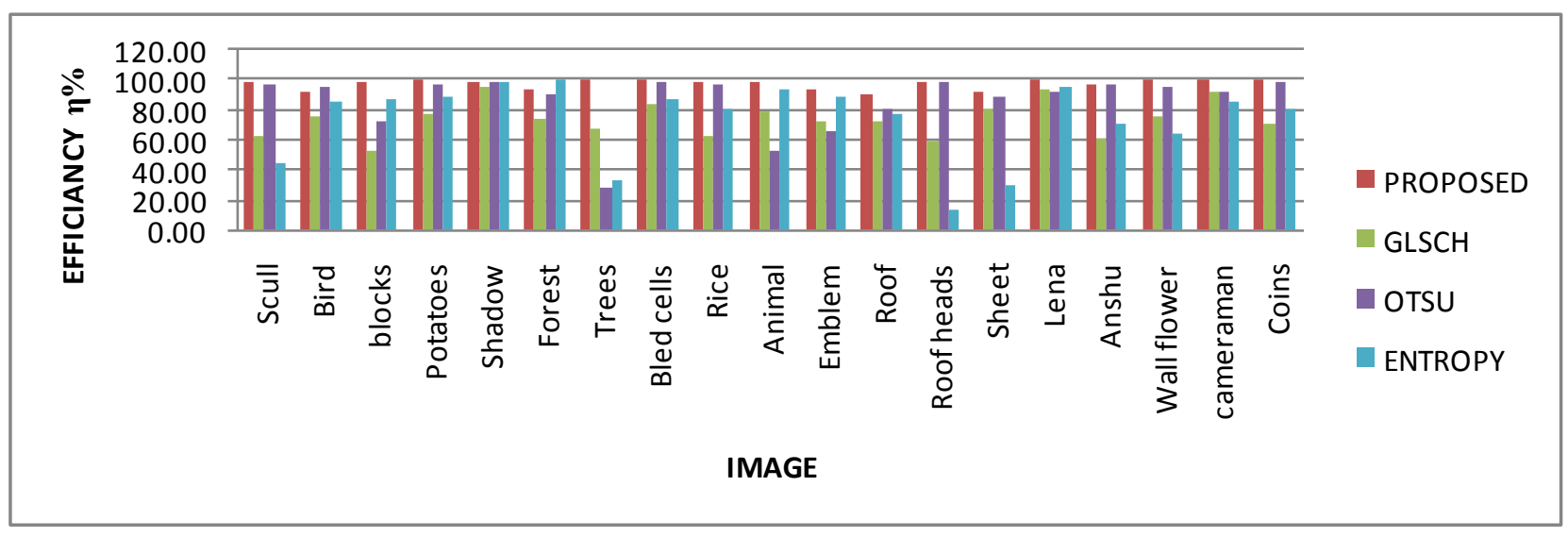

Fig 6: Efficiency of various thresholding techniques. 

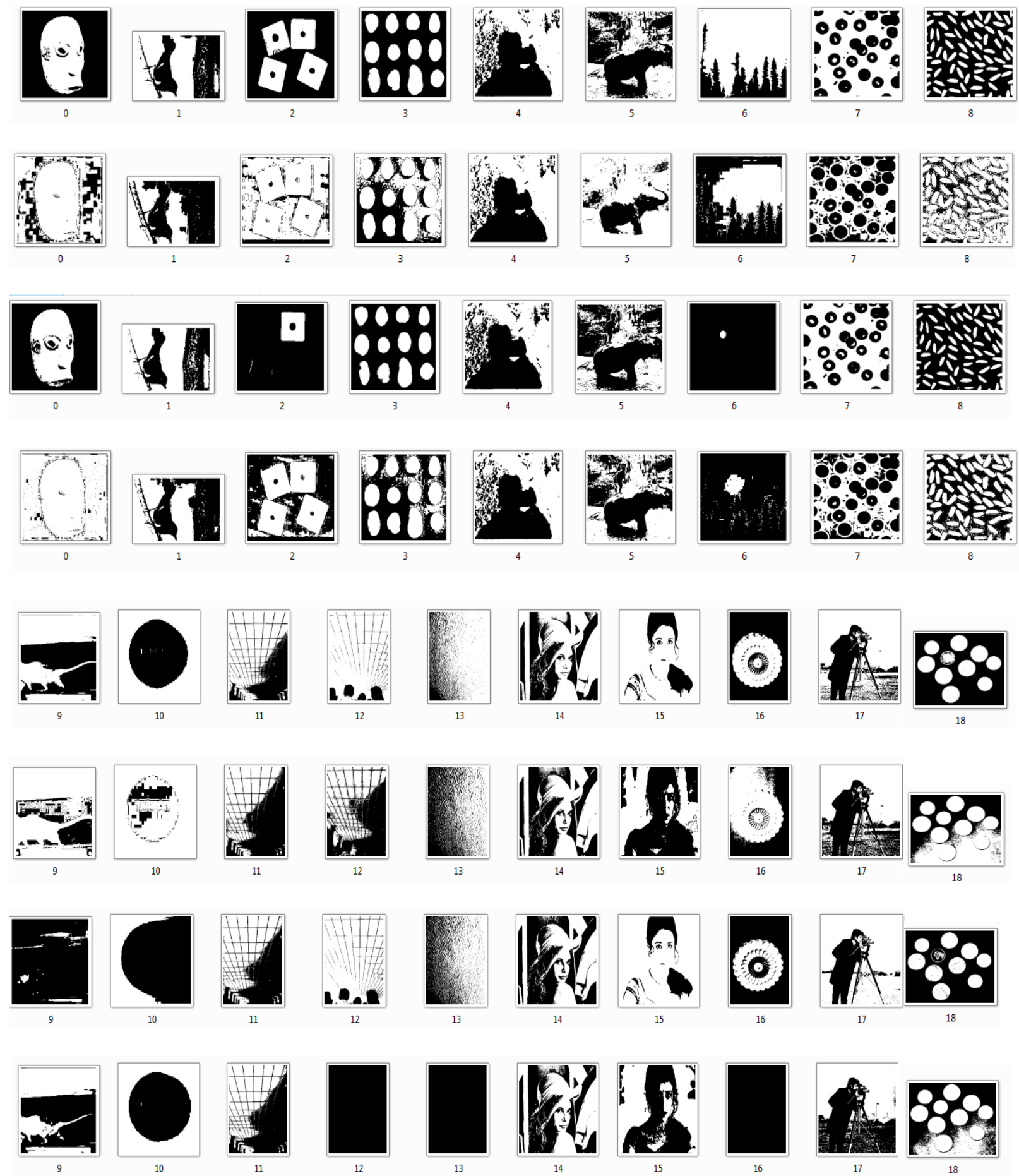

Fig 7: Results of four techniques from top to bottom proposed method, GLSCH entropy, Utso and Kapur's Entropy techniques.

\section{REFERENCES}

[1] R. C. Gonzalez and R. E.Woods, Digital Image Processing. Reading, MA: Addison-Wesley, 1993.

[2] M. Sezgin and B. Sankur, "Survey over image thresholding techniques and quantitative performance evaluation," $J$. Electron. Imag., vol. 13, no. 1, pp. 146-165, Jan. 2004.
[3] N. R. Pal and S. K. Pal, "A review on image segmentation techniques”, pattern recog.,vol.26,No. 9, pp.1277$1294,1993$.

[4] N. Otsu, "A threshold selection method from gray level histograms," IEEE Trans. Syst., Man, Cybern., vol. SMC-9, pp. 62-66, 1979. 
[5] C. E.Shannon,"A mathematical theory of communications", Bell. Sy st.,tech. pp.623-656,J.27,1948

[6] T. Pun, "A new method for gray-level picture thresholding using the entropy of the histogram," Signal Process., vol. 2, no. 3, pp. 223-237,1980.

[7] J. N. Kapur, P. K. Sahoo, and A. K. C.Wong, "A new method for gray level picture thresholding using the entropy of the histogram," Graph. Models Image Process., vol. 29, pp. 273-285, 1985.

[8] P.K. Sahoo, and G. Arora., "A thresholding method based on two-dimensional Renyi's entropy", Pattern Recognit., 2004, pp. 1149-1161.

[9] Sahoo, P., Willkins, C., and Yeager, J., "Threshold selection using Renyi’s entropy”, Pattern Recognit., 1997, pp. 71-84.
[10] P.K. Sahoo, and G. Arora, "Image thresholding using twodimensional Tsallis-Havrda-Charvát entropy", Pattern Recognit. Lett., 2006, pp. 520-528.

[11] Portes de Albuquerque, M., Esquef, I. A., et al., "Image thresholding using Tsall is entropy", Pattern Recognit. Lett., 2004 , pp. 1059-1065.

[12] Yang Xiao, Zhiguo Cao, Tianxu Zhang "Entropic thresholding based on gray level spatial correlation histogram",IEEE trans. $19^{\text {th }}$ international conf., pp. 14,ICPR-2008,

[13] A.S. Abutaleb, "Automatic thresholding of gray-level picture using two-dimensional entropies", Pattern Recognit., 1989, pp. 22-32. 